\title{
Study of microstructure and mechanical property on bulk Ni-Fe alloys fabricated by electrodeposition
}

\author{
Ding Wei \\ School of Material and Metallurgy, Inner Mongolia University of Science and Technology, Baotou \\ 014010, China \\ adingwei@126.com
}

Keywords: electrodeposition; Ni-Fe alloy; microstructure; mechanical property

\begin{abstract}
An electrodeposited Ni-Fe alloy with a nickel content of 75\% was introduced in this paper, thickness of this Ni-Fe alloy is $1 \mathrm{~mm}$. Microstructure, tensile mechanical, and hardness and the phase were studied by using field emission scanning electron microscopy (FE SEM), tensile test, hardness tester and X-ray diffraction (XRD). The result shows that the Fe-Ni alloy has a fine grain size and grain size is nanometer scale. It has a high tensile strength over $1500 \mathrm{Mpa}$ and has a high Vickers hardness (HV) which is 560, but it has not plasticity, its elongation rate is less than $1 \%$. By the technique of XRD, it can be found its alloy phase is $\mathrm{FeNi}_{3}$ of FCC structure.
\end{abstract}

\section{Introduction}

The Ni - Fe alloys have been widely considered for both scientific and practical applications due to their properties such as low thermal expansion, high magnetic permeability, excellent structural-quality and their mechanical properties. Various compositions of $\mathrm{Ni}-\mathrm{Fe}$ alloys such as, Permalloy (80Ni - 20Fe), Invar alloy (36Ni - 64Fe) and high strength $\mathrm{Ni}$ - Fe alloy can be used to produce microsensor, microactuators and other MEMS device[1].

Research about electrodeposited Ni-Fe alloys were focus on thin film Ni-Fe alloy, most of them thickness under $100 \mu \mathrm{m}$. Brook et al. investigated the effect of specimens' thickness on tensile ductility in electrodeposited nanocrystalline Ni-Fe alloys [2]. The results of their detailed study indicated that limited sample thickness $(<100 \mu \mathrm{m})$ could suppress tensile ductility. Recently, Isao Matsui, et al. reported an electrodeposited bulk nanocrystalline Ni-Fe alloys with high strength and high ductility[3].

Therefore, this parper aims to introduce a bulk Ni-Fe alloy fabricated by electrodeposition. While, microstructure and mechanical property of the Ni-Fe alloy were studied.

\section{Experiment process}

\subsection{The manufacturing of the Ni-Fe alloy}

To produce the nanometer scale $\mathrm{Ni}-\mathrm{Fe}$ (Ni $75 / \%$ and the rest of $\mathrm{Fe}$ ) block alloy by the method of electrodeposition. Its power is dc power, its positive pole is Ir oxide coated titanium plate, its negative pole is 304 stainless steel, its liquor is sulphate system whose temperature is about $(65 \pm 2){ }^{\circ} \mathrm{C}$ and the $\mathrm{PH}$ value of the solutions is about $3.0 \pm 0.2$. Electric current density is $2 \mathrm{~A} / \mathrm{dm}^{2}$ with compound liquor of pure regent and deionized water. The sample was washed by the deionized water after electrodeposited, then stripping the Fe-Ni alloy from stainless steel surfaces and drying. The size of the Ni-Fe alloy is $200 \mathrm{~mm} \times 200 \mathrm{~mm} \times 1 \mathrm{~mm}$.

\section{2 microstructure and mechanical properties test}

The room temperature tensile properties of the heat-treated samples were examined on a gauge length of $50 \mathrm{~mm}$ with a $12.5-\mathrm{mm}$ nominal width. Tensile tests were carried out in a SANS universal testing machine using a load range of $5000 \mathrm{kgf}$. The crosshead speed was maintained at $2 \mathrm{~mm} / \mathrm{min}$. Yield strength (Rel), tensile strength (Rm) and elongation (A50) were tested. Zess super55 type FE SEM can be used to observe surface morphology and tensile fracture of the Fe-Ni alloy. Using Rockwell hardness tester and Vickers tester respectively measures its macro-hardness and 
micro-hardness. X-ray diffractometry was carried out for crystalline preferred orientation. The crystallographic structure of the alloys was examined by X-ray diffraction (XRD) analysis with a $\mathrm{Cu}$ $\mathrm{K} \alpha$ source. The current and voltage were $40 \mathrm{~mA}$ and $50 \mathrm{kV}$, respectively. The registered angle range was $2 \theta=20-80^{\circ}$ with a step size $\Delta \mathrm{G} \theta=0.02^{\circ}$ and a measuring time of $1 \mathrm{~s}$ per step.

\section{Experimental results}

\subsection{Mechanical properties}

The tensile stress-strain curve of the Ni-Fe alloy is shown in Fig.1. It can be found the tensile strength reaches $1570 \mathrm{MPa}$, yield strength is $1280 \mathrm{MPa}$. There is no significant elongation and the elongation A50 less than 1\%. Using Rockwell hardness tester to measure its macro-hardness and the HRC is about 90. In view of the Rockwell hardness (HRC) commonly used range is between in 20-70, that the measured HRC value reflecting the Rockwell hardness number is real or not needs further discussion. In order to better reflect its hardness value, using Vickers hardness tester for this research to measure the micro-hardness value of Ni-Fe alloy. As shown in Fig.2 (b), HV value is about 560 while the value is 280 for the steel material of $800 \mathrm{MPa}$ tensile strength [4].

From the data of the Ni-Fe alloy tested on its mechanical properties, the alloy owns high strength and hardness but worse plasticity and no ductility. The alloy can't be used as structure alone, so how to take advantage of its hardness and strength is a worthy topic.

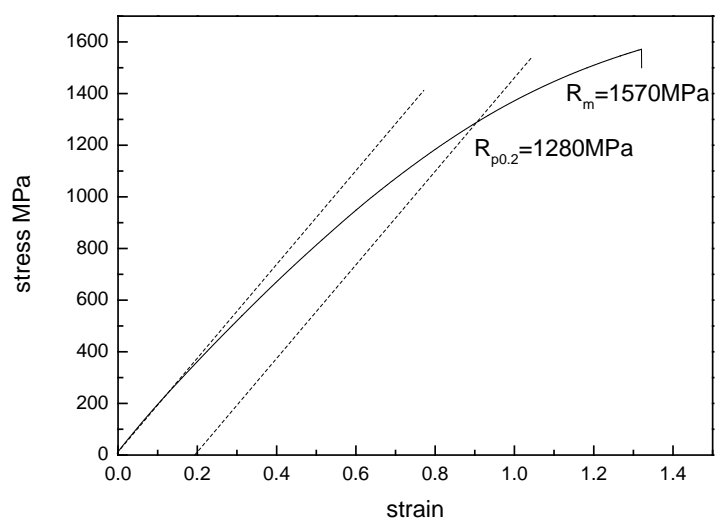

Fig.1 Tensile curve of stress and strain for Ni-Fe alloy
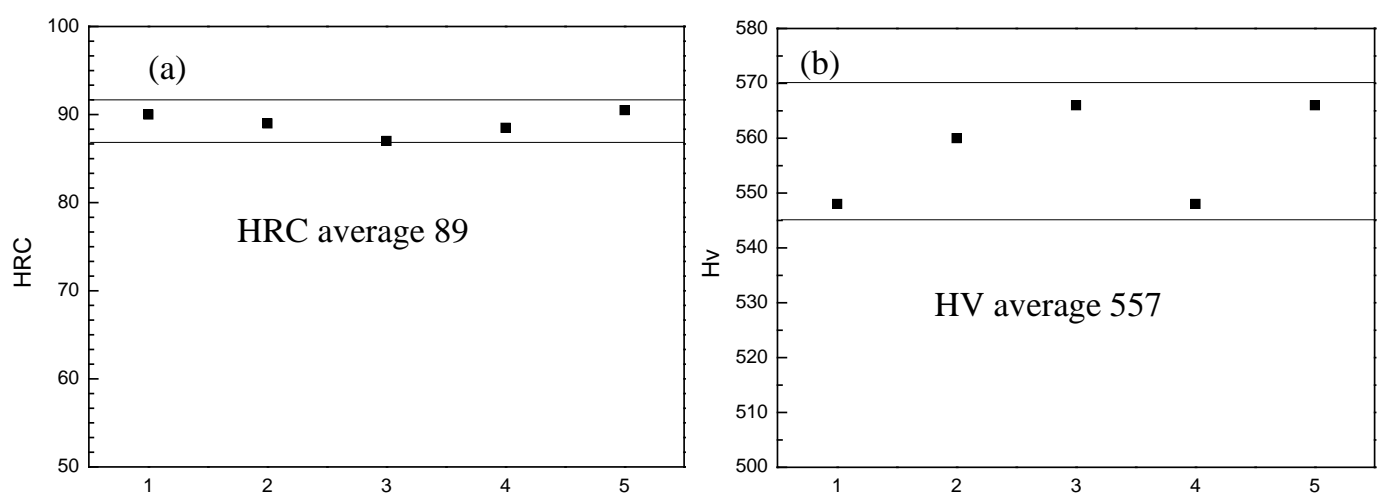

Fig.2 Hardness for Ni-Fe alloy (a) HRC; (b) HV

\subsection{Microstructure}

With the technique of Zess super55 FE SEM to observe the microstructure of Ni-Fe alloy, the result is shown in Fig.3. It can be found that the microstructure size of the Ni-Fe alloy is at nanometer scale, less than $200 \mathrm{~nm}$. So it can be found that the its microstructure appears clusters form whose growth direction perpendicular to the observation surface for the possible reason that it may exist 
preferred orientation in the process of growth of electro-deposition. In this paper, the microstructure needs to be a further study to clear its organizational characteristics.

The fracture scanning imagine of the Ni-Fe alloy tensile specimens is shown in Fig.4. The Fig.4 (a) shows the overall fracture appearance that presents a significant characteristic of brittle fracture. The fracture characteristics and the sample have nothing to do with the ductility. Also a few sockets appear on the fracture place that is shallow and bulky size but have no significant help to plastic deformation.

From the mechanical properties and scanning fracture organization aspects, its mechanical characteristics present a high strength and low plasticity characteristic. This may have something to do with its manufacturing. It uses the dc current to control production in this paper while literatures say that suing pulse current can improve its plasticity. In addition to other literature study, adjusting the composition of the proportion of $\mathrm{Fe}$ and $\mathrm{Ni}$ can also improve its plasticity.

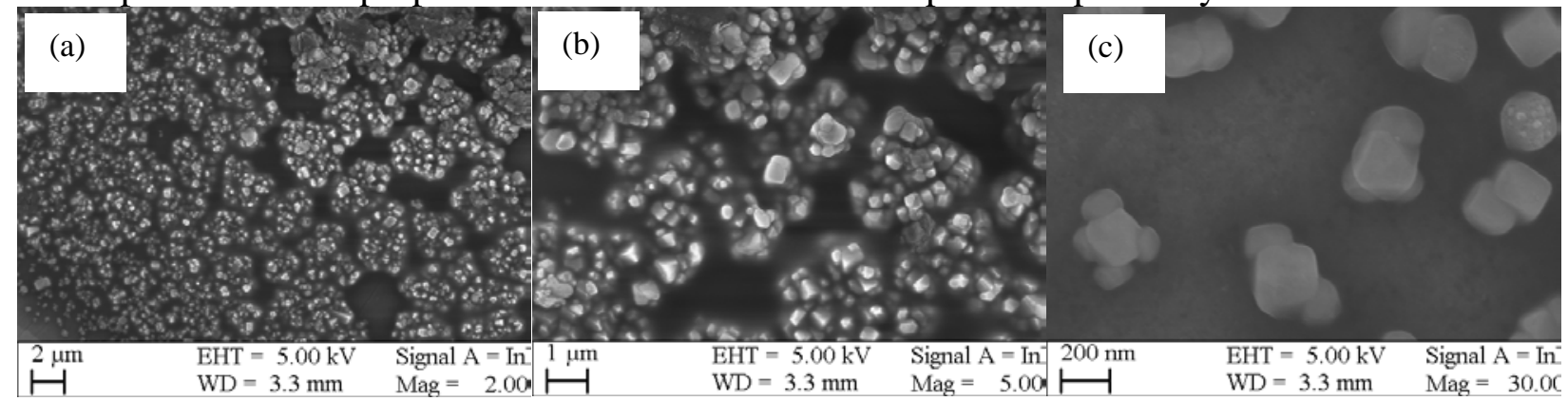

Fig.3 Microstructure of the Fe-Ni alloy

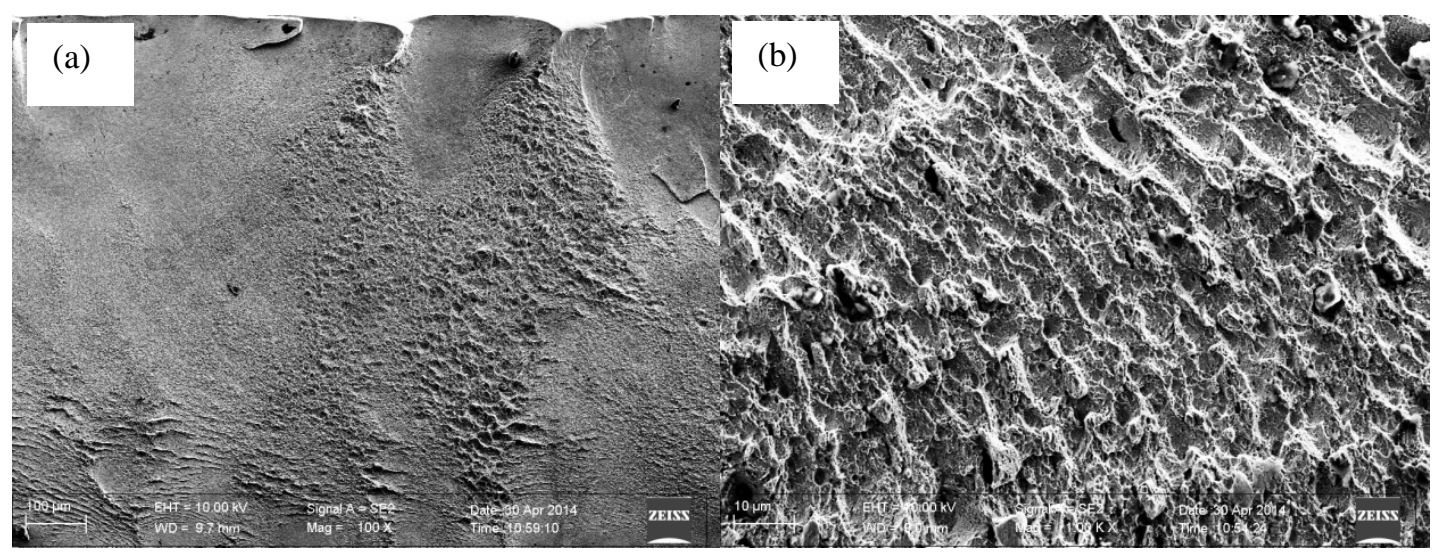

Fig.4 Tensile fracture analysis of the Fe-Ni alloy

\subsection{Phase analysis}

The XRD instrument is introduced in this paper to do the X-ray diffraction test for the Ni-Fe alloy, aiming to observe its structure shown in Fig.5.

Fig. 5 plots the XRD pattern of Ni-Fe alloys using microelectroforming process. As shown in Fig. 5, the electrodeposited Ni-Fe alloys have polycrystalline structure and a (1 111 ) preferred orientation peak is obvious. Mechanical properties of $\mathrm{Ni}-\mathrm{Fe}$ layer are related with preferred orientation. The different current densities and power supply styles used affected the crystallographic texture. Based on Cheung et al. [5] previous study, the intensity of $\left(\begin{array}{lll}2 & 0 & 0\end{array}\right)$ oriented peak decreased as Fe contents increased. The nanocrystallinization effect was also obvious with increasing Fe contents.

So, for the electrodeposition experiment of the Ni-Fe alloy, there is significantly preferred orientation in the (111) direction. Other literature reports that when the scanning angle increase to $100^{\circ}$, there also appear peaks in 91 and $97^{\circ}$ whose crystal structure is also face-centered cubic and its peak value lower than the $44^{\circ}$ [6]. Compared with the PDF card, the phase is determined as $\mathrm{FeNi}_{3}$.

By analyzing the XRD picture, the Ni-Fe alloy tested owns a face-centered crystal structure, and there is a significantly preferred orientation in the [111] direction in the electrodeposition experiment $[7,8]$. 


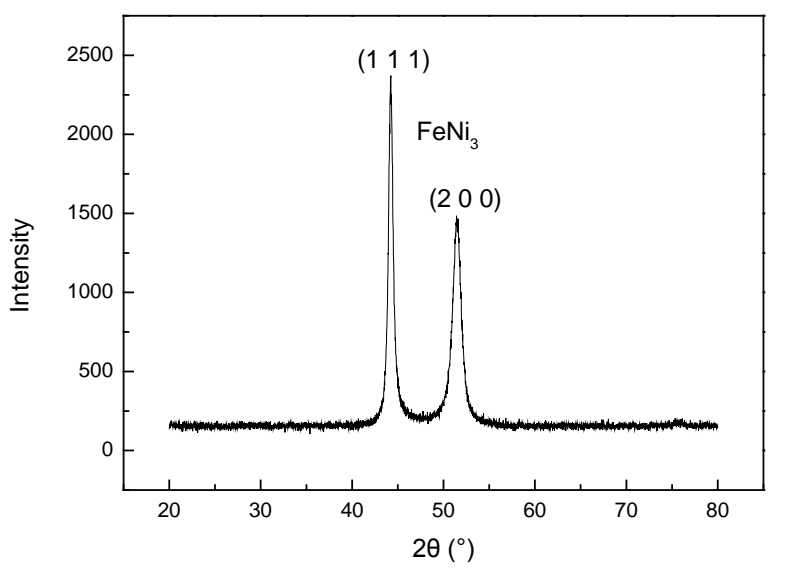

Fig.5 X-ray diffraction pattern of Ni-Fe alloy

\section{Conclusions}

The Ni-Fe bulk alloy owns high strength and hardness in this paper whose tensile strength reaches up to 1570Mpa and the HV value to 560 but no plasticity and little ductility. Also its structure appears clusters form and there maybe preferred orientation in the growth of the electrodeposition. And its phase is FeNi3 with FCC crystal structure.

\section{Acknowledgment}

This study was supported by Talent Incubation Funding of School of Materials and Metallurgy, IMUST (2014CY012).

\section{References}

[1] Y.-M Yeh, G.C Tu, T.-H Fang. Journal of Alloys and Compounds, Vol. 372 (2004), P.224

[2] Brooks I, Palumbo G,Hibbard G D,Wang ZR,Erb U.J Mater Sci, vol.21(2011),P.7713

[3] Isao Matsui , Tomo Kawakatsu,Yorinobu Takigawa,et al. Materials Letters, vol.116(2014),P.71

[4] Ding Wei. PHD paper, 2011

[5] C Cheung, F Djuanda, U Erb, G Palumbo. Nanostruct. Mater., 5 (1995), p. 513

[6] Yang Feng, Ma Li min, Zhou Shao-xiong, et al. Metallic Functional Materials.Vol.18(2011),p.6

[7] Gong Feng-fei, Kamel A, et al. Journal of East China Normal University (Natural Science), vol. Z1 (2005), P.63

[8]Yu Guanghua, Zhao Hongchen, Zhu Fengwu, et al. Vacuum science and technology(china), vol.21(2001),P.419 